\title{
Codes on fibre products of hyperelliptic curves*
}

\section{S. A. STEPANOV}

Abstract - The purpose of this paper is to construct a new family of smooth projective curves over a finite field $F_{q}$ with a lot of $F_{q}$-rational points. The genus in this family is considerably less than the number of rational points, so that the corresponding geometric Goppa codes have rather good parameters.

The work was supported by Bilkent University, 06533 Bilkent, Ankara, Turkey.

\section{INTRODUCTION}

Let $X$ be a smooth projective curve of genus $g=g(X)$ defined over a finite field $k^{\prime}=$ $F_{q}$. Recall the basic ideas of the Goppa construction [3] of the linear $[n, k, d]_{q}$-codes associated with the curve $X$. Let $\left\{x_{1}, \ldots, x_{n}\right\}$ be the set of $k^{\prime}$-rational points of $X$ and

$$
D_{0}=x_{1}+\ldots+x_{n} \text {. }
$$

Let $D$ be a $k^{\prime}$-rational divisor on $X$. We assume that $D$ has the support disjoint from $D_{0}$, i.e., the points $x_{i}, i=1, \ldots, n$, occur with multiplicity zero in $D$. Denote by $k^{\prime}(X)$ the field of rational functions on $X$ and consider the vector space over $k^{\prime}$

$$
L(D)=\left\{f \in k^{\prime}(X)^{*} \mid(f)+D \geq 0\right\} \cup\{0\} .
$$

The linear $[n, k ; d]_{q}$-code $C=C\left(D_{0}, D\right)$ associated with the pair $\left(D_{0}, D\right)$ is the image of the linear evaluation map

$$
\operatorname{Ev}: L(D) \rightarrow F_{q}^{n}, \quad f \mapsto\left(f\left(x_{1}\right), \ldots, f\left(x_{n}\right)\right) .
$$

Such a $q$-ary linear code is called a geometric Goppa code. If $\operatorname{deg} D<n$, then the map Ev is an injection, so that $C \simeq L(D)$.

Dually, denote by $\Omega(X)$ the $k^{\prime}(X)$-vector space of rational differential forms on $X$ and consider the linear space over $k^{\prime}$

$$
\Omega\left(D_{0}-D\right)=\left\{\omega \in \Omega(X)^{*} \mid(\omega)+D_{0}-D \geq 0\right\} \cup\{0\} .
$$

The linear map

$$
\operatorname{Res}: \Omega\left(D_{0}-D\right) \rightarrow F_{q}^{n}, \quad \omega \mapsto\left(\operatorname{Res}_{x_{1}}(\omega), \ldots, \operatorname{Res}_{x_{n}}(\omega)\right)
$$

${ }^{*}$ UDC 519.72. Originally published in Diskretnaya Matematika (1997) 9, No. 1, 83-94 (in Russian). Received May 12, 1996. Translated by the author. 
defines the linear $[n, k, d]_{q}$-code $C^{*}=C^{*}\left(D_{0}, D\right)$ associated with the pair $\left(D_{0}, D\right)$. If $\operatorname{deg} D>2 g-2$, then the map Res is injective, so that $C^{*} \simeq \Omega\left(D_{0}-D\right) \simeq L\left(K+D_{0}-D\right)$, where $K$ is a canonical divisor on $X$.

Each linear $[n, k, d]_{q}$-code $C$ defines a pair of its relative parameters $(\delta, R)$, where $\delta=d / n$ is the relative minimum distance and $R$ is the transmission rate of $C$. The points $(\delta, R)$ form the set of code points $V_{q}^{\text {lin }} \subseteq[0,1]^{2}$. Let $U_{q}^{\text {lin }}$ denote the subset of limit points of $V_{q}^{\text {lin }}$. In other terms, $(\delta, R) \in U_{q}^{\text {lin }}$ if and only if there exists an infinite sequence of different linear codes $C_{i}$ with relative parameters $\delta_{i}=\delta\left(C_{i}\right)$ and $R_{i}=R\left(C_{i}\right)$ such that

$$
\lim _{i \rightarrow \infty}\left(\delta_{i}, R_{i}\right)=(\delta, R) .
$$

If $\delta>0$ and $R>0$, then such a family of codes $C_{i}$ is called asymptotically good. The structure of $U_{q}^{\mathrm{lin}}$ can be described as follows (see [1, 4]): there exists a continuous function $\alpha_{q}^{\text {lin }}(\delta)$ such that

$$
U_{q}^{\text {lin }}=\left\{(\delta, R) \mid 0 \leq R \leq \alpha_{q}^{\operatorname{lin}}(\delta)\right\},
$$

moreover, $\alpha_{q}^{\operatorname{lin}}(0)=1, \alpha_{q}^{\text {lin }}(\delta)=0$ for $(q-1) / q \leq \delta \leq 1$, and $\alpha_{q}^{\text {lin }}(\delta)$ decreases on the interval $[0,(q-1) / q]$.

It follows from the Riemann-Roch theorem that the relative parameters $R=k / n$ and $\delta=d / n$ both for $L$ - and $\Omega$-constructions satisfy (see $[8,10]$ ) the inequality

$$
R \geq 1-\delta-\frac{g-1}{n}
$$

In order to produce a family of asymptotically good geometric Goppa codes for which $R+\delta$ comes above the Gilbert-Varshamov bound

$$
\alpha_{q}^{\operatorname{lin}}(\delta) \geq 1-H_{q}(\delta)
$$

where

$$
H_{q}(\delta)=\delta \log _{q}(q-1)-\delta \log _{q} \delta-(1-\delta) \log _{q}(1-\delta),
$$

one needs a family of smooth projective curves with a lot of $k^{\prime}$-rational points compared to the genus. Examples of such families are provided by classical modular curves $X_{0}(N)$ and $X(N)$ (see [5, 9]), or by Drinfeld modular curves (see [10], Chapters 4.1 and 4.2). Thus, if $q=p^{v}$ is an even power of a prime number $p$, then there exists an infinite sequence of geometric Goppa codes $C_{i}$ which gives the lower bound

$$
\alpha_{q}^{\operatorname{lin}} \geq 1-\delta-(\sqrt{q}-1)^{-1} .
$$

The line $R=1-\delta-(\sqrt{q}-1)^{-1}$ intersects the curve $R=1-H_{q}(\delta)$ for $q \geq 49$. Much easier proof of this result based on consideration of a sequence of (modified) Artin-Schreier coverings of the projective line $P^{1}\left(\bar{k}^{\prime}\right)$ was recently proposed by Garcia and Stichtenoth [2]. 
In this paper we consider a new family of smooth projective curves $X_{s}$ given over $k^{\prime}=F_{q}$ by equations

$$
z_{i}^{2}=f_{i}(u), \quad 1 \leq i \leq s,
$$

where $f_{i}(u)$ are relatively prime square-free polynomials in $k^{\prime}[u]$ of a special form. Every such curve is actually a fibre product of hyperelliptic curves. The main point of the paper is to calculate the genus $g\left(X_{s}\right)$ (Lemma 1) and determine the number $N_{q}\left(X_{s}\right)$ of $k^{\prime}$-rational points (Lemma 4) on the curve $X_{s}$. We show that the ratio $g\left(X_{s}\right) / N_{q}\left(X_{s}\right)$ is small enough and deduce from (1) that the corresponding geometric Goppa codes $C\left(D, D_{0}\right)$ and $C^{*}\left(D, D_{0}\right)$ have rather good parameters. For small values of $s$, these parameters are comparable with the parameters of codes on Artin-Schreier coverings introduced by Garcia and Stichtenoth [2]. In particular, if $s=1$, then the codes $C\left(D, D_{0}\right)$ and $C^{*}\left(D, D_{0}\right)$ have the same parameters as the codes on Hermitian curves (see [8], Section VII.3). Unfortunately, the parameter $s$ in our construction is bounded by $q^{1 / 2}$ and as a result the genus $g\left(X_{s}\right)$ is bounded by

$$
(q-3) 2^{\left(q^{1 / 2}-2\right)}+1
$$

However, since the above upper bound is large enough for $q \geq q_{0}$, the curves $X_{s}$ provide sufficiently long geometric Goppa codes.

A similar construction of non-singular projective curves with a lot of $k^{\prime}$-rational points based on the use of fibre products of some Artin-Schreier curves was independently considered by van der Geer and van der Vlugt [11].

The genus $g\left(X_{s}\right)$ can be easily calculated using the Hurwitz genus formula. However, we prefer to use a slightly more complicated argument which allows us to find explicitly a basis of the space $\Omega\left(D_{0}-D\right)$. This provides an easy way to write out the generator matrices for codes in the family and to find a fast decoding algorithm.

Applying to curves $X_{s}$ the Goppa constructions, we obtain the following results.

Theorem 1. Let $p>2$ be a prime number, $v>1$ be an even integer, and let $F_{q}$ be a finite field consisting of $q=p^{v}$ elements. For any positive integers $s \leq q^{1 / 2}$ and $l>\left(s q^{1 / 2}-3\right) 2^{s-2}$ there exists a geometric Goppa $[n, k, d]_{q}$-code $C=C\left(D_{0}, D\right)$ with parameters

$$
\begin{aligned}
& l<n \leq\left(2 q^{1 / 2}-s\right) q^{1 / 2} 2^{s-1} \\
& k \geq l-\left(s q^{1 / 2}-3\right) 2^{s-2} \\
& d \geq n-l
\end{aligned}
$$

Theorem 2. For $p, q$ and $s$ as in Theorem 1, and for any positive integer $l>$ $\left(s q^{1 / 2}-3\right) 2^{s-1}$ there exists a geometric Goppa $[n, k, d]_{q}$-code $C^{*}=C^{*}\left(D_{0}, D\right)$ with parameters

$$
\begin{aligned}
l-\left(s q^{1 / 2}-3\right) 2^{s-2} & <n \leq\left(2 q^{1 / 2}-s\right) q^{1 / 2} 2^{s-1}, \\
k & \geq n-l+\left(s q^{1 / 2}-3\right) 2^{s-2}, \\
d & \geq l-\left(s q^{1 / 2}-3\right) 2^{s-1} .
\end{aligned}
$$


Corollary 1. The relative parameters $R=k / n$ and $\delta=d / n$ of the above codes satisfy the inequality

$$
R \geq 1-\delta-\frac{\left(s q^{1 / 2}-3\right) 2^{s-2}}{n}
$$

In particular, for $n=\left(2 q^{1 / 2}-s\right) q^{1 / 2} 2^{s-1}$

$$
R \geq 1-\delta-\frac{s q^{1 / 2}-3}{2\left(2 q^{1 / 2}-s\right) q^{1 / 2}} .
$$

By a suitable concatenation one gets reasonably good codes over $F_{p}$. Indeed, let $k_{0}>1$ be an even number. Applying a linear $\left[n_{0}, k_{0}, d_{0}\right]_{p}$-code $C_{0}$ to an $[n, k, d]_{q}$-code $C=C\left(D_{0}, D\right)$ over $F_{q}$, where $q=p^{k_{0}}$, we obtain an $\left[n^{\prime}, k^{\prime}, d^{\prime}\right]_{p}$-code $C^{\prime}$ with parameters

$$
n^{\prime}=n_{0} n, \quad k^{\prime}=k_{0} k, \quad d^{\prime}=d_{0} d .
$$

Let us denote by $R_{0}=k_{0} / n_{0}$ and $\delta_{0}=d_{0} / n_{0}$ the relative parameters of the code $C_{0}$.

Corollary 2. For any positive integers $n_{0}>1, s \leq q^{1 / 2}$ and $l>\left(s q^{1 / 2}-3\right) 2^{s-2}$ there exists a linear $\left[n^{\prime}, k^{\prime}, d^{\prime}\right]_{p}$-code $C^{\prime}$ with parameters

$$
\begin{aligned}
n_{0} l & <n^{\prime}=n_{0} n \leq n_{0}\left(2 q^{1 / 2}-s\right) q^{1 / 2} 2^{s-1}, \\
k^{\prime} & \geq k_{0}\left(l-\left(s q^{1 / 2}-3\right) 2^{s-2}\right), \\
d^{\prime} & \geq d_{0}(n-l) .
\end{aligned}
$$

The relative parameters $R^{\prime}=k^{\prime} / n^{\prime}$ and $\delta^{\prime}=d^{\prime} / n^{\prime}$ of the code $C^{\prime}$ satisfy the inequality

$$
R^{\prime}+\delta^{\prime} \geq R_{0}\left(\frac{l}{n}-\frac{\left(s q^{1 / 2}-3\right) 2^{s-2}}{n}\right)+\delta_{0}\left(1-\frac{l}{n}\right) .
$$

Applying a linear $\left[n_{0}, k_{0}, d_{0}\right]_{p}$-code $C_{0}$ to a linear $[n, k, d]_{q}$-code $C^{*}=C_{*}\left(D_{0}, D\right)$, we obtain the following result.

Corollary 3. For any positive integers $n_{0}>1, s \leq q^{1 / 2}$ and $l>\left(s q^{1 / 2}-3\right) 2^{s-2}$ there exists a linear $\left[n^{\prime \prime}, k^{\prime \prime}, d^{\prime \prime}\right]_{p}$-code $C^{\prime \prime}$ with parameters

$$
\begin{aligned}
n_{0}\left(l-\left(s q^{1 / 2}-3\right)\right. & <n^{\prime \prime}=n_{0} n \leq n_{0}\left(2 q^{1 / 2}-s\right) q^{1 / 2} 2^{s-1}, \\
k^{\prime \prime} & \geq k_{0}\left(n-l+\left(s q^{1 / 2}-3\right) 2^{s-2}\right), \\
d^{\prime \prime} & \leq d_{0}\left(l-\left(s q^{1 / 2}-3\right) 2^{s-1}\right) .
\end{aligned}
$$

The relative parameters $R^{\prime \prime}=k^{\prime \prime} / n^{\prime \prime}$ and $\delta^{\prime \prime}=d^{\prime \prime} / n^{\prime \prime}$ of the code $C^{\prime \prime}$ satisfy the inequality

$$
R^{\prime \prime}+\delta^{\prime \prime} \geq R_{0}\left(1-\frac{l}{n}+\frac{\left(s q^{1 / 2}-3\right) 2^{s-2}}{n}\right)+\delta_{0}\left(\frac{l}{n}-\frac{\left(s q^{1 / 2}-3\right) 2^{s-1}}{n}\right) .
$$

The results of this paper can be extended to the case of fibre products of more general form over an arbitrary finite field. 


\section{NOTATION AND LEMMAS}

Let $k^{\prime \prime}$ be the algebraic closure of $k^{\prime}=F_{q}$ and $A^{s+1}$ be an $(s+1)$-dimensional affine space over $k^{\prime \prime}$. Assume that char $k^{\prime}>2$.

Lemma 1. Let $f_{1}, \ldots, f_{s}$ be pairwise coprime square-free monic polynomials in $k^{\prime}[u]$ of the same odd degree $m \geq 1$ and $Y$ be the fibre product in $A^{s+1}$ given over $k^{\prime}$ by the equations

$$
z_{i}^{2}=f_{i}(u), \quad 1 \leq i \leq s .
$$

Then the genus $g=g(X)$ of a smooth projective model $X$ of the curve $Y$ is equal to

$$
g=(m s-3) 2^{s-2}+1 .
$$

Proof. Let $X$ be a smooth projective model of the curve $Y$. Denote by $v_{x}$ the canonical valuation of the function field $k^{\prime \prime}(X)$, and by $\Omega[X]$ the space of regular differential forms on $X$. The affine curve $Y$ is easily seen to be smooth. If $\bar{Y}$ is its projective closure, then $X$ is a normalization of $\bar{Y}$ and we have the map $\psi: X \rightarrow \bar{Y}$ which is an isomorphism between $Y$ and $\psi^{-1}(\bar{Y})$. Hence it follows that $g=g(X)=g(Y)$.

The rational map $\left(u, z_{1}, \ldots, z_{s}\right) \mapsto u$ of the curve $Y$ in $A^{1}$ determines a morphism $\varphi: X \rightarrow P^{1}$ of degree $2^{s}$, so that for $u_{0} \in A^{1}$ either $\varphi^{-1}\left(u_{0}\right)$ consists of $2^{s}$ points of the form $x^{\prime}=\left(u_{0}, \pm z_{1}, \ldots, \pm z_{s}\right)$ at each of which $v_{x^{\prime}}(t)=1$ for the local parameter $t$ at $u_{0}$, or else $\varphi^{-1}\left(u_{0}\right)$ consists of $2^{s-1}$ points of the form $x_{i}^{\prime \prime}=\left(u_{0}, \pm z_{1}, \ldots \pm z_{i-1}, 0, \pm z_{i+1}, \ldots, \pm z_{s}\right)$, and $v_{x_{i}^{\prime \prime}}(t)=2$.

Let us consider the point at infinity $u_{\infty} \in P^{1}$. If the coordinate on $A^{1}$ is denoted by $u$, then $t=u^{-1}$ is the local parameter at $u_{\infty}$. If $\varphi^{-1}\left(u_{\infty}\right)$ consisted of $2^{s}$ points $x_{\infty}^{(\tau)}$, then at each $x_{\infty}=x_{\infty}^{(\tau)}$ the function $t$ would be the local parameter. Hence it would follow that $v_{x_{\infty}}(t)=1$ and $v_{x_{\infty}}\left(f_{i}(t)\right)=-m$. But since $m$ is odd, this contradicts the condition that $v_{x_{\infty}}\left(f_{i}(u)\right)=2 v_{x_{\infty}}\left(z_{i}\right)$. Thus, $\varphi^{-1}\left(u_{\infty}\right)$ consists of $r=2^{s-1}$ points $x_{\infty}^{(\tau)}, 1 \leq \tau \leq r$, with the projective coordinates $x_{\infty}^{(\tau)}=(0,1, \pm 1, \ldots, \pm 1,0)$. It follows that $X=Y \cup\left\{x_{\infty}^{(1)}\right\} \cup \ldots \cup\left\{x_{\infty}^{(r)}\right\}$. At any such point $x_{\infty}=x_{\infty}^{(\tau)}$ we have $v_{x_{\infty}}(u)=-2$ and $v_{x_{\infty}}\left(z_{i}\right)=-m$.

Let us now find a basis of the space $\Omega[X]$ over the field $k^{\prime \prime}$. Any element $\omega \in \Omega[Y]$ can be written as a $k^{\prime \prime}$-linear combination of the differential forms $\omega_{0}=P_{0}(u) d u$ and

$$
\omega_{i_{1}, \ldots, i_{\sigma}}=\frac{P_{i_{1}, \ldots, i_{\sigma}}(u) d u}{z_{i_{1}} \ldots z_{i_{\sigma}}}
$$

where $i_{1}, \ldots, i_{\sigma}$ are integers such that $1 \leq i_{1}<\ldots<i_{\sigma} \leq s$ and $P_{i_{1}, \ldots, i_{\sigma}}$ are polynomials in $k^{\prime \prime}[u]$. Indeed, the differential form

$$
\omega_{i_{1}, \ldots, i_{\sigma}}^{\prime}=\frac{d u}{z_{i_{1}} \ldots z_{i_{\sigma}}}
$$

is regular at any point $u_{0} \in A^{1}$ with the condition $z_{i}\left(u_{0}\right) \neq 0$ for $i \in\left\{i_{1}, \ldots, i_{\sigma}\right\}$. Now if $z_{i}\left(u_{0}\right)=0$ for an unique $i \in\left\{i_{1}, \ldots, i_{\sigma}\right\}$, then $z_{i}$ is the local parameter at 
$x_{i}^{\prime \prime}=\left(u_{0}, \pm z_{1}, \ldots, \pm z_{i-1}, 0, \pm z_{i+1}, \ldots, \pm z_{s}\right)$, so that $v_{x_{i}^{\prime \prime}}\left(z_{i}\right)=1$ and $v_{x_{i}^{\prime \prime}}\left(u-u_{0}\right)=2$. Therefore $v_{x_{i}^{\prime \prime}}(d u)=1$ and again $\omega_{i_{1}, \ldots, i_{\sigma}}^{\prime}$ is regular at $u_{0}$. The form $\omega_{0}^{\prime}=d u$ is also regular at any point $u_{0} \in A^{1}$. Thus, the differential forms $\omega_{0}^{\prime}=d u$ and $\omega_{i_{1}, \ldots, i_{\sigma}}^{\prime}$ form a basis of the $k^{\prime \prime}[u]$-module $\Omega[Y]$.

It remains to clarify which of the forms $\omega_{0}$ and $\omega_{i_{1}, \ldots, i_{\sigma}}$ are regular at the points $x_{\infty}^{(1)}, \ldots, x_{\infty}^{(r)}$. Let $x_{\infty}$ be one of these points. If $t$ is the local parameter at $x_{\infty}$, then $u=t^{-2} u^{\prime}, z_{i}=t^{-m} z_{i}^{\prime}$, where $u^{\prime}$ and $z_{i}^{\prime}$ are units in the local ring $O_{x_{\infty}}$. Therefore $\omega_{i_{1}, \ldots, i_{\sigma}}^{\prime}=t^{m \sigma-3} \eta_{i_{1}, \ldots, i_{\sigma}} d t$, where $\eta_{i_{1}, \ldots, i_{\sigma}}$ is a unit in $O_{x_{\infty}}$, hence $\left(\omega_{i_{1}, \ldots, i_{\sigma}}^{\prime}\right)=(m \sigma-3) x_{\infty}$. Thus, the differential form

$$
\omega_{i_{1}, \ldots, i_{\sigma}}=\frac{P_{i_{1}, \ldots, i_{\sigma}}(u) d u}{z_{i_{1}} \ldots z_{i_{\sigma}}}
$$

is regular at $x_{\infty}$ if and only if

$$
v_{x_{\infty}}\left(P_{i_{1}, \ldots, i_{\sigma}}(u)\right) \geq-(m \sigma-3) .
$$

This means that $\operatorname{deg} P_{i_{1}, \ldots, i_{\sigma}}(u) \leq(m \sigma-3) / 2$ and hence

$$
\operatorname{deg} P_{i_{1}, \ldots, i_{\sigma}}(u) \leq \begin{cases}(m \sigma-4) / 2, & \text { if } \sigma \equiv 0(\bmod 2), \\ (m \sigma-3) / 2, & \text { if } \sigma \equiv 1(\bmod 2) .\end{cases}
$$

The differential form $\omega_{0}=P_{0} d u$ is not regular at $x_{\infty}$ for any non-zero polynomial $P_{0} \in k^{\prime \prime}[u]$, therefore the regular differential forms

$$
\omega_{i_{1}, \ldots, i_{\sigma}}^{\prime}, \quad u \omega_{i_{1}, \ldots, i_{\sigma}}^{\prime}, \quad, u^{n} \omega_{i_{1}, \ldots, i_{\sigma}}^{\prime},
$$

where $1 \leq i_{1}<\ldots<i_{\sigma} \leq s$ and

$$
n= \begin{cases}(m \sigma-4) / 2, & \text { if } \sigma \equiv 0(\bmod 2) \\ (m \sigma-3) / 2, & \text { if } \sigma \equiv 1(\bmod 2),\end{cases}
$$

form a basis of the space $\Omega[X]$ over $k^{\prime \prime}$. Therefore

$$
\begin{aligned}
\operatorname{dim}_{k^{\prime \prime}} \Omega[X] & =\frac{1}{2} \sum_{\sigma \equiv 0} \sum_{(\bmod 2)}(m \sigma-2)+\frac{1}{2} \sum_{\sigma \equiv 1} \sum_{(\bmod 2)}\left(m \sigma-\ldots<i_{\sigma} \leq s\right. \\
& =\frac{m}{2} \sum_{\sigma=1}^{s} \sigma\left(\begin{array}{l}
s \\
\sigma
\end{array}\right)-\sum_{\sigma \equiv 0(\bmod 2)}\left(\begin{array}{l}
s \\
\sigma
\end{array}\right)-\frac{1}{2} \sum_{\sigma \equiv 1(\bmod 2)}\left(\begin{array}{l}
s \\
\sigma
\end{array}\right) \\
& =\frac{1}{2}\left(m s 2^{s-1}-2^{s}-2^{s-1}+2\right)
\end{aligned}
$$

and hence

$$
g=g(X)=\operatorname{dim}_{k^{\prime \prime}} \Omega[X]=(m s-3) 2^{s-2}+1 .
$$

This completes the proof. 
Let $p$ be a prime number, $v$ be a positive integer and let $F_{q}$ be a finite field with $q=p^{v}$ elements. The field $F_{q}$ is a Galois extension of the prime finite field $F_{p}$ of degree $v$ with the cyclic Galois group of order $v$. The action of a generator $\theta$ of this group on an element $x \in F_{q}$ is given by the rule $\theta(x)=x^{p}$. The map

$$
\operatorname{norm}_{v}(x)=x \theta(x) \ldots \theta^{v-1}(x)=x x^{p} \ldots x^{p^{v-1}}
$$

of $F_{q}$ onto $F_{p}$ is the norm of the element $x$.

Let $\chi$ be a multiplicative character of the field $F_{p}$ and $x$ an element of $F_{q}$. Set

$$
\chi_{v}(x)=\chi\left(\operatorname{norm}_{v}(x)\right)
$$

and call $\chi_{v}$ a multiplicative character of the field $F_{q}$ induced by the character $\chi$.

Now let $f$ be a square-free polynomial in the ring $F_{q}[u]$ of degree $m$ and let $\chi$ be a non-trivial quadratic character of $F_{p}$. Consider the character sum

$$
S_{v}(f)=\sum_{u \in F_{q}} \chi_{v}(f(u))=\sum_{u \in F_{q}} \chi\left(\operatorname{norm}_{v}(f(u))\right)
$$

and recall the well-known Weil bound [12] (see also [7], Chapters 1 and 5)

$$
\left|S_{v}(f)\right| \leq 2\left[\frac{m-1}{2}\right] q^{1 / 2} .
$$

The following result of the author (see [6], Theorem 3 ) shows us that the Weil bound cannot be sharpened essentially in any extension $F_{q}$ of the field $F_{p}$.

Lemma 2. Let $F_{q}$ be a finite field with $q=p^{v}$ elements of characteristic $p>2$ and let $\chi_{v}$ be the character of $F_{q}$ induced by a non-trivial quadratic character $\chi$ of the field $F_{p}$. If $v>1$, then for the square-free polynomial $f \in F_{p}[u]$,

$$
f(u)= \begin{cases}u+u^{p^{v / 2}}, & \text { if } v \equiv 0(\bmod 2), \\ \left(u+u^{p^{(v-1) / 2}}\right)\left(u+u^{p^{(v+1) / 2}}\right), & \text { if } v \equiv 1(\bmod 2),\end{cases}
$$

we have

$$
\sum_{u \in F_{q}} \chi_{v}(f(u))= \begin{cases}\left(q^{1 / 2}-1\right) q^{1 / 2} & \text { if } v \equiv 0(\bmod 2) \\ q-1 & \text { if } v \equiv 1(\bmod 2)\end{cases}
$$

Proof. Let $v>1$ be an even number. Since $u^{p^{v}}=u$ in $F_{q}$, for any $u \in F_{q}$ we have

$$
\begin{aligned}
\operatorname{norm}_{v}(f(u)) & =\prod_{i=1}^{v}\left(u+u^{p^{v / 2}}\right)^{p^{i-1}}=\prod_{i=1}^{v}\left(u^{p^{i-1}}+u^{p^{v / 2+i-1}}\right) \\
& =\prod_{i=1}^{v / 2}\left(u^{p^{i-1}}+u^{p^{v / 2+i-1}}\right) \prod_{j=1}^{v / 2}\left(u^{p^{v / 2+j-1}}+u^{p^{j-1}}\right) \\
& =\prod_{i=1}^{v / 2}\left(u^{p^{i-1}}+u^{p^{v / 2+i-1}}\right)^{2} .
\end{aligned}
$$


Therefore

$$
\sum_{u \in F_{q}} \chi_{v}(f(u))=\sum_{u \in F_{q}} \chi\left(\operatorname{norm}_{v}(f(u))\right)=q-N,
$$

where $N$ is the number of elements of the set $A=\left\{u \in F_{q} \mid f(u)=0\right\}$. Since $f(u)=u\left(1+u^{p^{v / 2}-1}\right)$, we have $A=\{0\} \cup B$, where

$$
B=\left\{u \in F_{q} \mid 1+u^{p^{1 / 2}-1}=0\right\}
$$

is the set of roots of the polynomial $1+u^{p^{v / 2}-1}$ in $F_{q}$. Taking into account that the greatest common divisor of $\left(p^{v / 2}-1\right.$ and $\left.p^{v}-1\right)$ is equal to $p^{v / 2}-1$, we obtain from the Euler criterion that the number of roots of the polynomial $1+u^{p^{v / 2}-1}$ is equal to $p^{v / 2}-1$. In that case

$$
N=|A|=1+|B|=1+\left(p^{v / 2}-1\right)=q^{1 / 2},
$$

and hence

$$
\sum_{u \in F_{q}} \chi_{v}(f(u))=\left(q^{1 / 2}-1\right) q^{1 / 2}
$$

This proves the lemma for $v$ an even positive integer.

Let now $v>1$ be an odd number. In this case for any $u \in F_{q}$

$$
\begin{aligned}
\operatorname{norm}_{v}(f(u)) & =\prod_{i=1}^{v}\left(u^{p^{i-1}}+u^{p^{(v-1) / 2+i-1}}\right)\left(u^{p^{i-1}}+u^{p^{(v+1) / 2+i-1}}\right) \\
& =\prod_{i=1}^{(v-1) / 2}\left(u^{p^{i-1}}+u^{p^{(v-1) / 2+i-1}}\right) \prod_{i=(v+1) / 2}^{v}\left(u^{p^{i-1}}+u^{p^{(v+1) / 2+i-1}}\right) \\
& \times \prod_{i=1}^{(v-1) / 2}\left(u^{p^{i-1}}+u^{p^{(v+1) / 2+i-1}}\right) \prod_{i=(v+1) / 2}^{v}\left(u^{p^{i-1}}+u^{p^{(v+1) / 2+i-1}}\right) \\
& =\prod_{i=1}^{(v+1) / 2}\left(u^{p^{i-1}}+u^{p^{(v-1) / 2+i-1}}\right) \prod_{j=1}^{(v-1) / 2}\left(u^{p^{(v+1) / 2+j-1}}+u^{p^{j-1}}\right) \\
& \times \prod_{i=1}^{(v-1) / 2}\left(u^{p^{i-1}}+u^{p^{(v+1) / 2+i-1}}\right) \prod_{j=1}^{(v+1) / 2}\left(u^{p^{(v-1) / 2+j-1}}+u^{p^{j-1}}\right) \\
& =\prod_{i=1}^{(v+1) / 2}\left(u^{p^{i-1}}+u^{p^{(v-1) / 2+i-1}}\right)^{2} \prod_{i=1}^{(v-1) / 2}\left(u^{p^{i-1}}+u^{p^{(v+1) / 2+i-1}}\right)^{2}
\end{aligned}
$$

and hence

$$
\sum_{u \in F_{q}} \chi_{v}(f(u))=\sum_{u \in F_{q}} \chi\left(\operatorname{norm}_{v}(f(u))\right)=q-N^{\prime},
$$

where $N^{\prime}$ is the cardinality of the set $A=\left\{u \in F_{q} \mid f(u)=0\right\}$. Clearly, $N^{\prime}=1$ and therefore

$$
\sum_{u \in F_{q}} \chi_{v}(f(u))=q-1
$$

This completes the proof. 
Lemma 3. Let $F_{p}$ be a prime finite field of characteristic $p>2, F_{q}=F_{p^{v}}$ be an extension of $F_{p}$ of even degree $v>1$ and let $A$ be the set of roots in $F_{q}$ of the polynomial

$$
f(u)=u+u^{p^{1 / 2}}
$$

Then

(i) A is a subgroup of the additive group $F_{q}^{+}$of the field $F_{q}$;

(ii) if $\left\{A_{1}=A, A_{2}, \ldots, A_{r}\right\}$ is the set of all cosets in $F_{q}^{+} / A$ and $\left\{\alpha_{1}, \alpha_{2}, \ldots, \alpha_{r}\right\}$ are distinct representatives of the cosets, then the polynomials

$$
f_{i}(u)=\left(u+\alpha_{i}\right)+\left(u+\alpha_{i}\right)^{p^{v / 2}}, \quad 1 \leq i \leq r
$$

are pairwise coprime in $F_{q}[u]$;

(iii) $r=\left|F_{q}^{+} / A\right|=p^{v / 2}$.

Proof. The main point is (i). First of all we note that $f(0)=0$. Now if $\alpha$ and $\beta$ are zeros of $f(u)$, then

$$
\begin{aligned}
f(\alpha+\beta) & =(\alpha+\beta)+(\alpha+\beta)^{p^{v / 2}}=\alpha+\alpha^{p^{v / 2}}+\left(\beta+\beta^{p^{v / 2}}\right) \\
& =f(\alpha)+f(\beta)=0
\end{aligned}
$$

so that $\alpha+\beta$ is also a root of the polynomial $f(u)$. Thus, $A$ is a subgroup of $F_{q}^{+}$.

To prove (ii), let us suppose that $f_{i}(u)$ and $f_{j}(u)$ for $i \neq j$ have a common root in $F_{q}$, say $u=\theta$. In that case

$$
\theta+\alpha_{i}+\left(\theta+\alpha_{i}\right)^{p^{v / 2}}=\theta+\alpha_{j}+\left(\theta+\alpha_{j}\right)^{p^{v / 2}}
$$

and therefore

$$
\theta+\alpha_{i}+\theta^{p^{v / 2}}+\alpha_{i}^{p^{v / 2}}=\theta+\alpha_{j}+\theta^{p^{v / 2}}+\alpha_{j}^{p^{v / 2}}
$$

This yields

$$
\alpha_{i}-\alpha_{j}+\left(\alpha_{i}-\alpha_{j}\right)^{p^{p / 2}}=0
$$

and we find that $\alpha_{i}-\alpha_{j}$ is a root of $f(u)$, hence $\alpha_{i}-\alpha_{j} \in A$. But $\alpha_{i}-\alpha_{j} \notin A$ according to the choice of $\alpha_{1}, \ldots, \alpha_{r}$, and we arrive at a contradiction.

Finally, since $|A|=p^{v / 2}$, we find that

$$
r=\left|F_{q}^{+} / A\right|=p^{v} / p^{v / 2}=p^{v / 2} .
$$

This completes the proof. 
Lemma 4. Let $F_{p}$ be a prime finite field of characteristic $p>2, F_{q}$ be an extension of $F_{p}$ of even degree $v>1$ and let $s \leq q^{1 / 2}$ be a positive integer. Let $N_{q}$ be the number of $F_{q}$-rational points of the affine curve $Y$ given by equations $(2)$ with the polynomials

$$
f_{i}(x)=\left(u+\alpha_{i}\right)+\left(u+\alpha_{i}\right)^{p^{v / 2}}, \quad 1 \leq i \leq s,
$$

defined by (3). Then

$$
N_{q}=\left(2 q^{1 / 2}-s\right) q^{1 / 2} 2^{s-1}
$$

Proof. We have

$$
\begin{aligned}
N_{q} & =\sum_{u \in F_{q}}\left(1+\chi_{v}\left(f_{1}(u)\right)\right) \ldots\left(1+\chi_{v}\left(f_{s}(u)\right)\right) \\
& =\sum_{u \in F_{q}}\left(1+\sum_{\sigma=1}^{s} \sum_{1 \leq i_{1}<\ldots<i_{\sigma} \leq s} \chi_{v}\left(f_{i_{1}}(u)\right) \ldots \chi_{v}\left(f_{i_{\sigma}}(u)\right)\right)
\end{aligned}
$$

and hence

$$
N_{q}=p^{v}+\sum_{\sigma=1}^{s} \sum_{1 \leq i_{1}<i_{2}<\ldots<i_{\sigma} \leq s s} \sum_{u \in F_{q}} \chi_{v}\left(f_{i_{1}}(u)\right) \ldots \chi_{v}\left(f_{i_{\sigma}}(u)\right) .
$$

It follows from Lemmas 2 and 3 that

$$
\chi_{v}\left(f_{i}(u)\right)= \begin{cases}0, & \text { if } u \in A_{i}, \\ 1, & \text { if } u \in F_{q} \backslash A_{i},\end{cases}
$$

and since any two distinct sets $A_{i}$ and $A_{j}$ have no common element, we obtain

$$
\begin{aligned}
N_{q} & =p^{v}+\sum_{\sigma=1}^{s}\left(\begin{array}{l}
s \\
\sigma
\end{array}\right)\left(p^{v}-\sigma p^{v / 2}\right)=p^{v}+\left(2^{s}-1\right) p^{v}-s 2^{s-1} p^{v / 2} \\
& =\left(2 p^{v / 2}-s\right) p^{v / 2} 2^{s-1}=\left(2 q^{1 / 2}-s\right) q^{1 / 2} 2^{s-1} .
\end{aligned}
$$

This proves the lemma.

\section{PROOF OF THE THEOREMS}

Let $p>2$ be a prime number, $k^{\prime}=F_{q}$ be an extension of a prime finite field $F_{p}$ of an even degree $v>1$, and let $s \leq q^{1 / 2}$ be a positive integer. Let $f_{1}, \ldots, f_{s}$ be pairwise coprime polynomials in $k^{\prime}[u]$ of the same degree $q^{1 / 2}$ defined by (3), and let $Y \subset A^{s+1}$ be the affine curve defined over $k^{\prime}$ by equations (2). Let $\bar{Y} \subset P^{s+1}$ be the projective closure of $Y$, and $X$ be a non-singular projective model of $\bar{Y}$ over the algebraic closure $k^{\prime \prime}$ of the field $k^{\prime}$.

Since the curves $\bar{Y}$ and $X$ are birationally isomorphic, we have $g=g(Y)=g(X)$, and by Lemma 1

$$
g=\left(s q^{1 / 2}-3\right) 2^{s-2}+1
$$


Next, let $N_{q}$ be the number of $k^{\prime}$-rational points of $Y$ and $M_{q}$ be the number of $k^{\prime}$-rational points of $X$. We have $M_{q} \geq N_{q}+1$, and by Lemma 4

$$
M_{q} \geq\left(2 q^{1 / 2}-s\right) q^{1 / 2} 2^{s-1}+1
$$

Let $n \leq N_{q}$ be a positive integer, let $x_{1}, \ldots, x_{n}$ be $k^{\prime}$-rational points of the curve $X$ at the finite part of $X$, and let $x_{\infty}$ be the point of $X$ at infinity. Set

$$
D_{0}=x_{1}+\cdots+x_{n}, \quad D=l x_{\infty} .
$$

Applying to $X$ the $L$-construction for $l>\left(s q^{1 / 2}-3\right) 2^{s-2}$ and $n>l$, we obtain the geometric Goppa $[n, k, d]_{q}$-code $C=C\left(D_{0}, D\right)$ with parameters

$$
\begin{aligned}
& l<n \leq\left(2 q^{1 / 2}-s\right) q^{1 / 2} 2^{s-1}, \\
& k \geq l-g+1=l-\left(s q^{1 / 2}-3\right) 2^{s-2}, \\
& d \geq n-l .
\end{aligned}
$$

This proves Theorem 1.

Now, applying to $X$ the $\Omega$-construction for

$$
l>\left(s q^{1 / 2}-s\right) q^{1 / 2} 2^{s-1}, \quad n>l-\left(s q^{1 / 2}-3\right) 2^{s-2},
$$

we obtain the geometric Goppa $[n, k, d]_{q}$-code $C^{*}=C^{*}\left(D_{0}, D\right)$ with parameters

$$
\begin{aligned}
l-\left(s q^{1 / 2}-3\right) 2^{s-2} & <n \leq\left(2 q^{1 / 2}-s\right) q^{1 / 2} 2^{s-1}, \\
k & \geq n-l+\left(s q^{1 / 2}-3\right) 2^{s-2}, \\
d & \geq l-\left(s q^{1 / 2}-3\right) 2^{s-1} .
\end{aligned}
$$

This gives the result of Theorem 2 .

Finally, it follows from (1) that the relative parameters $R=k / n$ and $\delta=d / n$ of the codes $C=C\left(D_{0}, D\right)$ and $C^{*}=C^{*}\left(D_{0}, D\right)$ satisfy the inequality

$$
R \geq 1-\delta-\frac{\left(s q^{1 / 2}-3\right) 2^{s-2}}{n}
$$

This proves Corollary 1 . 


\section{REFERENCES}

1. M. J. Aaltonen, Notes on the asymptotic behavior of the information rate of block codes. IEEE Trans. Inform. Theory (1984) 30, 84-85.

2. A. Garcia and H. Stichtenoth, A tower of Artin-Schreier extensions of function fields attaining the Drinfeld-Vladut bound. Invent. Math. (1995) 121, 211-222.

3. V. G. Goppa, Codes on algebraic curves. Soviet Math. Dokl. (1981) 24, 170-172.

4. Yu. I. Manin, What is the maximum of points on a curve over $F_{2}$ ? J. Fac. Sci. Tokyo (1981) 28, 715-720.

5. C. Moreno, Algebraic Curves over Finite Fields. Cambridge Univ. Press, Cambridge, 1991.

6. S. A. Stepanov, On lower bounds of character sums over finite fields. Discrete Math. Appl. (1992) 2, 523-532.

7. S. A. Stepanov, Arithmetic of Algebraic Curves. Plenum, New York, 1994.

8. H. Stichtenoth, Algebraic Function Fields and Codes. Springer, Berlin, 1993.

9. M. A. Tsfasman, S. G. Vladut, and Th. Zink, Modular curves, Shimura curves, and Goppa codes, better than the Varshamov-Gilbert bound. Math. Nachr. (1982) 109, 21-28.

10. M. A. Tsfasman and S. G. Vladut, Algebraic-Geometric Codes. Kluwer Acad. Publ., Dordrecht, 1991.

11. G. van der Geer and M. van der Vlugt, Fibre products of Artin-Schreier curves and generalized Hamming weights of codes. J. Comb. Theory (1995) 70A, 337-348.

12. A. Weil, Number of solutions of equations in finite fields. Bull. Amer. Math. Soc. (1949) 55, 497-508. 